\title{
La educación de las ciencias, la Historia de la Ciencia y el libro de texto, las condiciones necesarias contra las suficientes
}

\section{J. Bruce Brackenbridge}

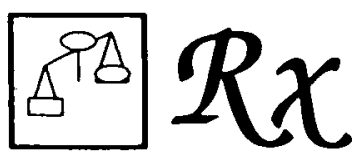

Según el autor, la dependencia del libro de texto, alta en todas las materias, es mayor en el caso de las ciencias. Pero su papel debe estar controlado y enriquecido por fuentes que permitan transmitir la visión bistórica: única que puede contextualizar el conocimiento y traer el texto a la vida.

La distinción más visible entre la enseñanza de una disciplina científica como la Física y la enseñanza de una disciplina humanística como la Historia es la total dependencia por parte de la primera de un libro de texto. El estudio de la Historia puede emplear un texto, pero continuamente se hace referencia tanto por parte del texto como del instructor a fuentes primarias. Los cursos de Historia en Colegios y Universidades apoyan el texto con alguna forma de estas fuentes básicas. Por el contrario, el físico localiza un «buen» libro de texto para poder impartir un curso autosuficiente, una situación que domina en la educación de las ciencias desde el Instituto hasta bien entrada la Universidad.

Hay, por supuesto, referencias a autoridades ajenas al libro de texto en la educación de las ciencias. En cierto sentido, la prueba experimental es la fuente primaria de la ciencia. Pero se entiende que el libro de texto ha revisado fielmente para el alumno esa fuente primaria además de acomodarla dentro de un esquema conceptual y teórico. Como en la versión final el libro de texto es autónomo, las referencias al pasado normalmente aparecen sólo en el primer capítulo del libro. Salvo referencias ocasionales a nombres y fechas, no vuelven a aparecer. Y aunque esto ocurra, el alumno descubre pronto que raramente aparecen en el examen. Lo importante es aprender a aplicar las reglas y las leyes tal como se expresan en el texto a los grupos de problemas que aparecen al final de los capítulos.

El papel preponderante del libro de texto en la educación de las ciencias lo ha tratado con detalle considerable Thomas Kuhn en The Structure of Scienti- 
fic Revolutions (La estructura de las revoluciones científicas). Este librito, publicado en 1962 por un joven físico convertido en historiador y filósofo de la ciencia, ha alcanzado nivel de difusión más elevado que cualquier otra obra similar, incluidas las de autores tales como Popper y Lakatos. Aunque su difusión académica no ha supuesto su aceptación universal como nuevo dogma de «lo que es la ciencia», sí que ha supuesto que el libro rara vez sea ignorado. Ya sea elogiado con entusiasmo o contestado con fuerza, está calro que The Structure of Scientific Revolutions ofrece por igual a defensores y detractores una visión fresca, incisiva y llena de sentido de esa actividad compleja llamada ciencia. Y una clave importante para entender esta visión de la ciencia reside en el papel que Kuhn asigna al libro de texto en la educación científica.

\section{EL PAPEL DEL LIBRO DE TEXTO DENTRO DE LA CIENCIA NORMAL}

La clave para entender la obra de Kuhn es biográfica. Al desarrollar su tesis hace referencias constantes a «información autobiográfica». En principio se educó como físico y, a partir de un análisis de su proceso de adiestramiento, produjo sus primeras observaciones originales de un modelo de «ciencia normal». Después, tras doctorarse en Física, fue a Harvard, donde James B. Conant le introdujo a la historia de la ciencia y «... de este modo inicié la transformación de (mi) concepción de la naturaleza del avance científico». Este interés por la Historia de la Ciencia dio pie en 1957 a su libro The Copernican Revolution ( $\mathrm{La}$ revolución copernicana). Después, Kuhn hizo su aparición como filósofo profesional de la ciencia en 1962 con la publicación de The Structure of Scientific Revolutions.

\section{Sentido global y restringido de «paradigma»}

Esta sucesión fuertemente unida de intereses por la Física, la Historia de la Ciencia y la Filosofía de la Ciencia proporcionó a Kuhn observaciones sobre la naturaleza de la ciencia nada obvias para el historiador sin conocimientos de Física o al físico sin conocimientos de Historia. El concepto clave de Kuhn, «paradigma», se compone de dos elementos: uno obvio para el físico y otro obvio para el historiador. Esta combinación es a la vez solución y fuente de los problemas que tiene Kuhn con sus críticos (ver Brackenbridge, 1985, p. 434). En palabras de Kuhn:

«El concepto de paradigmas probó ser el elemento perdido que requería para escribir el libro (The Structure of Scientific revolutions)... Desgraciadamente, en el proceso, los paradigmas tomaron vida propia... Habiendo empezado simplemente como soluciones ejemplares a los problemas (la tradición del libro de texto, obvia para el físico), ampliaron su imperio para incluir primero a los libros clásicos donde aparecieron inicialmente estos ejemplos aceptados y finalmente al conjunto total de compromisos compartidos por los miembros de una comunidad científica particular (la perspectiva histórica, obvia para el historiador). Este uso más globalizador del término es el único que han reconocido la mayoría de los lectores del libro, y el resultado inevitable ha sido la confusión» (1977, pp. 14-20).

En la posdata de la segunda edición de The Structure of Scientific Revolutions (1970), Kuhn establece su distinción entre los dos elementos de su concepto de paradigma. EL «uso» más «globalizador del término» se llamaría «matriz disciplinar» y el uso más restringido se llamaría «grupo de ejemplos». La «ma- 
triz disciplinaria» global se refiere a lo que los practicantes de una disciplina en particular tienen en común, y se llama «matriz» porque se compone de elementos ordenados de varias clases. Kuhn da «generalizaciones simbólicas», «partes metafísicas» y «valores compartidos» como ejemplos de estos elementos. $\mathrm{Ni}$ es una lista completa ni siquiera puede cerrarse tal lista, por un lado porque es un grupo abierto y por otro porque algunos de los elementos no pueden articularse directamente. Este sentido global de paradigma se refiere a la perspectiva histórica muy importante en el aprendizaje del historiador. Kuhn afirma que la mayoría de los lectores han reconocido sólo este sentido global y que por esto algunos críticos afirman que ha aportado poco que sea nuevo. Es, por lo tanto, en el sentido restringido de paradigma, el grupo de ejemplos, donde debe buscarse la contribución de Kuhn.

El sentido restringido de paradigma es obvio para el físico del mismo modo que se le escapa al humanista. La contribución de Kuhn es mostrar que proporciona una visión dentro de una estructura más amplia de la ciencia. Dicho simplemente, la Física es lo que los físicos hacen, y los físicos hacen lo que se les enseña a hacer. Es más, a los físicos se les enseña con libros de texto. La comprensión por parte de Kuhn de la tradición del libro de texto en la Física le condujo al concepto de paradigma y a sus observaciones iniciales dentro de la estructura total de la Física. Establece claramente su primer esfuerzo para desarrollar esta comprensión en una introducción autobiográfica a su posterior colección de ensayos, The Essential Tension (La tensión esencial):

«El esfuerzo requerido... es autobiográfico. Y mis esfuerzos a veces me han llevado a sentir que mi vida intelectual anterior pasaba ante mis ojos.» $(1977$, p. 10).

En un intento de establecer lo que llamaría «ciencia normal», Kuhn quiso especificar el consenso que identificaba a los miembros de una comunidad científica. Se volvió hacia el libro de texto como fuente de «acuerdo sobre las características definitorias de términos cuasi-teóricos tales como «fuerza» y «masa»» (Kuhn, 1977, p. 18).

Pero econtró que los físicos rara vez insistían sobre tales definiciones y, cuando esto ocurría, a menudo diferían fuertemente uno de otro.

«Me di cuenta ya en 1959 que no se requería un consenso de esa clase. A los científicos se les enseñaba, si no definiciones, sí maneras típicas de resolver problemas escogidos en los que figuraban términos como «fuerza» o «compuesto». Aceptando un grupo suficiente de estos ejemplos típicos, podrían dirigir su propia ivestigación a partir de ellos sin necesidad de acordar qué grupo de características de estos ejemplos los convertían en típicos... Los libros de texto exigen soluciones concretas a problemas que la profesión ha venido a aceptar como paradigmas» (p. 19).

De este humilde principio, como problemas de libro de texto, surge el sentido restringido de paradigma. Pero hay que fijarse en que el papel que Kuhn asigna a estos grupos de ejemplos va mucho más allá de su papel tradicional, que es la prueba de que el alumno comprende el texto. A los ojos de Kuhn, los ejemplos contienen y transmiten visiones de los principios de la física que no pueden articularse directamente en los capítulos del libro de texto.

Los sentidos restringido y global de paradigma están claramente relacionados. En la posdata a la segunda edición de The Structure of Scientific Revolucions, Kuhn describe el grupo de ejemplos como el elemento central único de la matriz disciplinar para el cual «el término "paradigma" sería completamente adecuado, tanto en el sentido filológico como autobiográfico» (1970, pp. 186-187). 
De este modo, paradigma en sentido restringido es realmente parte de paradigma en sentido global; una relación que Kuhn, en un tono más bien de disculpa, dice que «ha causado dificultades innecesarias a muchos lectores» (1977, p. 19). El libro de texto entonces se coloca en el centro del grupo de ejemplos, que a su vez se coloca en el centro de la matriz disciplinar, que a su vez es «el grupo global de compromisos compartidos por los miembros de una comunidad científica particular».

\section{CIENCIA NORMAL}

Con lo que más a menudo se identifica a Kuhn es con la idea de «revoluciones de la ciencia». Pero el concepto «paradigma» nace a partir de su forcejeo para poder describir la «ciencia normal», y es esa descripción de ciencia normal su principal contribución a nuestra visión de la naturaleza de la ciencia. La idea de revolución científica ni es nueva en Kuhn ni tampoco él afirma que lo sea. Muchos historiadores y filósofos han tratado hechos tales como la revolución copernicana mucho antes de la aparición del libro de Kuhn. Lo que es nuevo en la perspectiva de Kuhn es la naturaleza de la ciencia normal que cambia con esa revolución.

En el segundo capítulo de The Structure of Scientific Revolutions Kuhn describe «El Camino hacia la Ciencia Normal», indicando hasta qué punto el paradigma (en sentido global) depende del libro de texto:

«Si un científico concreto da por supuesto un paradigma, ya no necesitará partir de cero en sus obras fundamentales, comenzando con principios básicos y justificando el uso de cada concepto que se introduce. Esto puede quedar para el autor de libros de texto. Sin embargo, dado un libro de texto, el científico creativo puede iniciar su investigación donde termina la de aquél y de este modo concentrarse exclusivamente en los aspectos más sutiles y esotéricos de los fenómenos naturales que interesan a su grupo" (1970, pp. 19-20).

En el tercer capítulo, «Naturaleza de la Ciencia Normal», Kuhn establece las categorías de las actividades experimentales y teóricas que realizan los que se ocupan de la ciencia normal. Pero es el cuarto capítulo, «La Ciencia Normal como Resolución de Enigmas», el que da la visión más útil de lo que es la ciencia a los ojos de Kuhn. Rechaza la visión baconiana de una ciencia que busca abiertamente lo inesperado y afirma en su lugar que el rasgo de los problemas normales de la investigación que más interés despierta es que «no se dirigen a la novedad inesperada» (p. 35). En este sentido, los problemas de la ciencia normal comparten características importantes con enigmas de tipo «rompecabezas» $\mathrm{y}$ «damero».

«Aunque el resultado pueda preverse, a menudo con tanto detalle que lo que quede por conocer carezca de interés, el modo de llegar a ese resultado es en gran parte dudoso... Entonces el reto (del científico normal) es la convicción de que tan sólo con la habilidad suficiente conseguirá resolver un enigma que nadie había resuelto o resuelto así de bien» (pp. 36-38).

En el adiestramiento del científico como solucionador de enigmas, el libro de texto alcanza su cenit. Como ya se ha dicho, el libro de texto es eficiente en establecer la base del científico contemporáneo y permitirle «concentrarse exclusivamente en los aspectos más sutiles y esotéricos de los fenómenos naturales». Es efectivo al emplear ejemplos en forma de enigmas resueltos para adies- 
trarlo. De este modo le enseña al joven científico los tipos de problemas a los que debe acercarse, así como las técnicas que deben emplear al resolverlos. Y finalmente, el libro de texto es indispensable por suministrar precisamente el modo por el cual se comunican los científicos. «Los mismos libros de texto pretenden comunicar el vocabulario y la sintaxis del lenguaje científico contemporáneo» (p. 136).

\section{EL PAPEL DEL LIBRO DE TEXTO ENTRE CIENCIAS NORMALES}

El párrafo final del capítulo octavo de The Structure of Scientific Revolutions anuncia un cambio en el tema del libro: «... la transición resultante hacia un nuevo paradigma es una revolución científica, un tema que por fin estamos preparados para acometer directamente» (p. 90). En otras palabras, la primera mitad del libro se dedica a tratar de la "ciencia normal» y sólo sobre la base de ese concepto está preparado Kuhn a tratar las revoluciones. En el capítulo noveno trata de «Naturaleza y Necesidad de las Revoluciones Científicas» y en el décimo presenta a las «Revoluciones como Cambio del Concepto del Mundo», un capítulo que se abre con la afirmación de que «tras una revolución, los científicos reaccionan ante un mundo diferente» (p. 111) y se cierra con la afirmación «tras una revolución, los científicos trabajan en un mundo diferente» ( $\mathrm{p}$. 135).

Pero en el capítulo 11, «La Invisibilidad de las Revoluciones», afirma que los ejemplos que ha escogido «acostumbran a ser vistos no como revoluciones, sino como adiciones al conocimiento científico» (p. 136). ¿Cómo es posible que algo dramático como la creación de un «nuevo mundo» permanezca «invisible»?

«Yo diría que hay razones sobresalientes para que las revoluciones resulten ser casi invisibles. Tanto científicos como profanos se hacen una idea de la actividad científica creativa principalmente a partir de una fuente con autoridad que sistemáticamente disfraza - en parte por importantes razones de tipo funcional-- la existencia y significado de las revoluciones científicas. Sólo si se reconoce y analiza la naturaleza de esa autoridad se puede esperar conceder todo su efecto al ejemplo histórico... Como fuentes de autoridad me vienen a la cabeza principalmente los libros de texto de ciencias, así como los trabajos divulgativos y filosóficos hechos a su imagen» (p. 136).

Así pues, a los ojos de Kuhn el libro de texto «disfraza sistemáticamente» la historia de su disciplina. Afirma que «en parte por selección y en parte por distorsión» a los científicos de una época previa «se los representa implícitamente trabajando sobre el mismo grupo de problemas fijos y de acuerdo con el mismo grupo de cánones fijos» de los científicos contemporáneos. Es una tendencia que Kuhn describe como «escribir la historia hacia atrás» y que produce una ciencia de libro de texto que parece ser principalmente acumulativa más que revolucionaria. Y es una tendencia difícil de evitar si tras una revolución el científico vive en un mundo diferente. El libro de texto acaba contando la historia como debería haver sido, no necesariamente como fue. Además de ser éstos los modos eficiente y efectivo de presentar el material, el libro de texto debe contarlo así porque el autor desconoce a estas alturas lo que ocurría en el pasado. En palabras de Kuhn:

«El desprecio del dato histórico está arraigado profundamente, y probablemente por motivos funcionales, en la ideología de la profesión científica, la misma profesión que contempla como el mayor de los valores unos hechos de otras clases» (p. 138). 


\section{EL PAPEL DE LA HISTORIA EN LA EDUCACION DE LA CIENCIA}

Dado pues el papel central y crítico del libro de texto en la educación científica y dado que presenta distorsionada la historia de su disciplina, las preguntas que dedan son tres: (1) ¿Es posible corregir la situación? (2) ¿Es deseable corregir la situación? (3) y si es posible y deseable, ¿procede corregir la situación? Dicho de otro modo, el libro de texto tal como existe ahora es necesario para la educación científica pero, ¿es suficiente?

Como ejemplo de la tradición por parte de los libros de texto de emplear «historia escrita hacia atrás» para adoctrinar a los jóvenes científicos eficiente y efectivamente en la física contemporánea, se puede ver cómo se evalúan las contribuciones de Aristóteles, Galileo y Newton en el desarrollo de la cinemática y la dinámica. Es un tema central de todo libro de introducción a la física en todo Instituto y Universidad, que empieza normalmente por el tratamiento de movimientos en caída libre acerca de la superficie de la Tierra: Newton y la manzana. En este cuento, Newton desarrolla el prototipo del cálculo y lo aplica a problemas en la forma de su segunda ley, $\mathrm{F}=$ ma. Se le ve como partiendo de la obra de Galileo, el cual se ve a su vez demostrando tal movimiento dejando caer dos bolas distintas desde la torre inclinada de Pisa. El libro de texto atribuye a Galileo una demostración experimental clara del principio de que la relación con que caen libremente los cuerpos es independiente de su peso. La oposición a este hallazgo se atribuye a los escritos de Aristóteles, y a la Iglesia y al estamento universitario, que seguían a ciegas estos escritos. Esta visión popular de la situación la resume un físico conocido internacionalmente en un libro de texto introductorio del tipo «física para poetas».

«Aristóteles afirmaba que los cuerpos caen en proporción directa a su peso. Era evidente para todos que esto no era cierto. Sin embargo, nadie se atrevía a contradecir a Aristóteles hasta que llegó Galileo. Y él (Galileo) no era muy querido.»

Alguna versión de esta afirmación se repite en la mayoría de los libros de texto, a menudo de una forma ligeramente más sofisticada. El punto importante y esencial que el alumno debe asimilar del libro de texto es que si la fuerza gravitatoria $\mathrm{F}$ cerca de la superficie de la tierra es igual a $\mathrm{mg}$, donde $\mathrm{g}$ es $9,8 \mathrm{~m} / \mathrm{s}^{2}$, la aceleración a de la segunda ley de Newton, $\mathrm{F}=\mathrm{ma}$, es igual a la constante $\mathrm{g}$ y por tanto es independiente de la masa $\mathrm{m}$. Pero como este resultado importante puede relacionarse con las grandes figuras históricas de Newton, Galileo y Aristóteles, es difícil que el libro de texto resista la tentación de contar la historia a la vez que establece este punto, particularmente cuando Aristóteles y Newton parecen diferir dramáticamente en el mismo.

En un esfuerzo de evaluar la afirmación de Kuhn de que tras una revolución el libro de texto vuelve a escribir la historia, es de provecho comparar los escritos mismos de estas figuras históricas con los escritos que el libro de texto les atribuye. Un tratamiento anterior, aunque deficiente, del fondo de la cuestión de la caída libre se encuentra en el libro de Lane Cooper, Aristotle, Galileo and the Tower of Pisa (1936) (Aristóteles, Galileo y la Torre de Pisa). Como profesor de lengua y literatura, Lane Cooper consideró un reto la afirmación típica de libro de texto de Física de que Aristóteles había mantenido una postura equivocada «evidentemente». Preguntó a sus colegas de la Facultad de Física sobre los resultados obvios del experimento de la torre de Pisa y averiguó que ninguno de ellos lo había hecho, pero que creían que, de realizarlo, el experimento resultaría según decía el libro de texto. Entonces Cooper buscó en los escritos 
de Aristóteles su afirmación sobre la caída libre, pero no la encontró. Ningún libro de texto daba una referencia de la fuente de la afirmación de Aristóteles.

El tema que tratan Aristóteles, Galileo y Newton es el movimiento en la nada, es decir, una investigación teórica de cinemática y dinámica idealizadas. Es este rasgo, que Aristóteles llama la nada y que el libro de texto llama el vacío, el que une los argumentos. Aristóteles recoge este tema en el libro cuarto de una obra llamada Física A ojos modernos, parece ser una obra de Filosofía de la ciencia más que de las ciencias físicas. Sin embargo, contiene un argumento sobre el movimiento en el cual un cuerpo más pesado se mueve más rápido que un cuerpo más ligero. Es más, la estructura del argumento es paralela al tratamiento de la obra de Aristóteles que Galileo refiere en Las dos nuevas ciencias.

Es central en la visión del mundo físico por parte de Aristóteles la afirmación de que la nada no puede existir. Hay una larga historia de la nada en la tradición presocrática de la ciencia griega, pero basta simplemente con ver este argumento de Aristóteles en particular. Establece el problema como sigue:

«Vemos un cuerpo de peso determinado que se mueve en proporción más rápida o más lenta por una o dos razones: o, por una parte, a causa de una diferencia en el medio, como por ejemplo tierra, agua o aire; o, por otra, a causa de una diferencia en los cuerpos móviles comparados de tal modo que, siendo iguales otras cosas, un cuerpo es más ligero o más pesado que el otro» (Aristóteles, 1961, 215a20, p. 72).

A este argumento parece referirse Galileo en Las dos nuevas ciencias cuando establece la postura de Aristóteles sobre los cuerpos en caída:

«Aristóteles lucha contra algunos antiguos que introdujeron la nada como necesaria para el movimiento, diciendo que el movimiento no podría existir sin ella. Aristóteles, en oposición a esto, prueba que, al contrario, la existencia del movimiento, el cual vemos, destruye el supuesto de la nada; y éstos son sus pasos: Hace dos suposiciones: Una referente a móviles distintos en peso pero moviéndose en el mismo medio y la otra referente a un móvil dado moviéndose en medios distintos» (Galileo, 1974,106, p. 65).

El contexto y lo que sigue deja claro que Galileo se refiere al movimiento uniformemente acelerado tal como ocurre en la «caída desde una altura». Por lo tanto es provechoso examinar cuidadosamente la situación física que trata Aristóteles. Trata, como afirma Galileo, del movimiento de un cuerpo dado en medios que ofrecen distinta resistencia y el movimiento de cuerpos distintos en un medio dado. A partir del primer punto, Aristóteles infiere que, si la nada existe, un cuerpo atravesará un trecho de la nada y un trecho igual de cierta no-nada al mismo tiempo. De aquí, como tal la situación es contraria a la lógica o a la observación, concluye que la nada no puede existir. Del segundo punto deduce «que, siendo otras cosas iguales, los cuerpos pesados y ligeros se mueven con velocidades desiguales, en un espacio igual, en la proporción que sus magnitudes tienen entre sí» (Aristóteles, 1961, 216215, p. 74). Y, por lo visto, esta es la afirmación que Galileo y el libro de texto encuentran ser falsa «obviamente».

Pero Aristóteles no trata cuerpos en caida libre ni trata movimiento acelerado de ningún tipo. A través de toda la exposición, a un cuerpo único se le da una velocidad característica en cada medio y en un medio único se da a cada cuerpo una velocidad única pero distinta en cada caso. Lo que más se aproxima a esta situación es la de los cuerpos que caen con velocidad final en un medio que ofrece resistencia, el llamado problema de Stokes. El libro de texto lo trata a menudo como el movimiento de esferas en el que el «freno» se supone ser 
proporcional a la velocidad, $v$, y la velocidad final se alcanza cuando la fuerza de freno, bv (donde b es una constante), es igual a la fuerza gravitatoria constante, $\mathrm{mg}$. De este modo, la velocidad final o característica es igual a $\mathrm{mg} / \mathrm{b}$ y de este modo la proporción en la caída es directamente proporcional al peso $\mathrm{mg}$ (para un $b$ dado). «Los cuerpos pesados y ligeros se mueven con velocidades desiguales en un espacio igual en la proporción que sus magnitudes tienen entre sí.» Se diría, por lo tanto, que Aristóteles está más «en lo cierto, obviamente» que «equivocado, obviamente».

Se debe tener el mismo cuidado en no atribuir el «movimiento de Stokes» al razonamiento de Aristóteles que en aceptar la atribución de Galileo de que se trata de movimiento en caída libre. Sin embargo, lo que debería estar claro es que si es «obvio» para el libro de texto que los cuerpos en la nada no caerían en proporción directa a su peso, también debería ser «obvio» para una mente de primer orden como Aristóteles que fuera así. Y una lectura atenta de las fuentes primarias indica que la situación no era tan simple como la historia reescrita del libro de texto la presentaba. Se diría, por lo tanto, que el propio Galileo es culpable de escribir la historia hacia atrás en su libro de texto las dos nuevas ciencias al afirmar que Aristóteles se refería a la caída libre. Galileo hace que su portavoz, Sagrado, cite directamente a Aristóteles, aunque son éstas las palabra que Lane Cooper no encuentra en ningún pasaje aristotélico:

«Sagrado: dice Aristóteles, "una bola de hierro de cien libras que cae desde la altura de cien brazas llega al suelo antes de que una de sólo una libra ha descendido una braza". Yo (Galileo) digo que llegan al mismo tiempo. Encontrarás al hacer el experimento que la más grande se adelanta a la más pequeña en dos pulgadas, es decir, cuando la grande golpea el suelo, la otra está a dos pulgadas por detrás de ella. Y ahora quieres ocultar tras estas dos pulgadas las noventa y nueve brazas de Aristóteles, y hablando sólo de mi pequeño error permaneces callado ante el suyo enorme» (Galileo, 1974, 109, p. 68).

Qué afirmación más noble. Una apelación al experimento y a la prueba empírica, una extrapolación de lo real a lo ideal y hasta un fundamento para hablar del error experimental. Desgraciadamente, Lane Cooper describe la afirmación como «invento puro por parte de Galileo de unas palabras puestas en boca de Aristóteles» (Cooper, 1936, p. 52). Sin embargo, ¿qué importa que Aristóteles no dijese eso de verdad? Ciertamente debe ser lo que muchos defensores de Aristóteles contemporáneos de Galileo creían que dijo.

Sin embargo, después de la afirmación sobre lo experimental, Galileo continúa con un hermoso argumento teórico a partir de una modificación del experimento imaginado por Aristóteles en la Física. En contraste con Aristóteles, Galileo considera dos cuerpos moviéndose en dos medios distintos que ofrecen resistencia. En cada caso, el cuerpo más pesado sí cae más rápido que el más ligero, pero la diferencia es menor en el medio que opone menor resistencia: a menor densidad de medio, menos diferencia en su movimiento. Cuando uno extrapola el movimiento a una nada hipotética, el pesado y el ligero caen con la misma proporción. Esta extensión del argumento bien podía pensar Galileo que hasta Aristóteles la reconocería como proceso.

Pero hay que recordar el punto inicial de Aristóteles. Simplemente, que no es posible que la nada exista en la naturaleza. Galileo no discute esa afirmación directamente, más bien argumenta que es posible inferir el movimiento relativo de dos cuerpos distintos como si estuvieran en la nada a partir de la extrapolación de su movimiento relativo en una serie de medios distintos. Con un criterio postnewtoniano se puede alegar que la aceptación por parte de Galileo de 
una nada hipotética producirá más fruto que el rechazo por parte de Aristóteles de una nada hipotética producirá más fruto que el rechazo por parte de Aristóteles de una nada real. Sin embargo, a la mayoría de los libros de texto no les basta este criterio, es más, llegan a concluir que Aristóteles no consigue apreciar la importancia de la observación en la ciencia.

Hay que considerar hasta qué punto la ciencia posterior al siglo XVII ha rendido tributo a los métodos experimentales y teóricos expuestos por Newton en sus Principia. El libro de texto alaba su afirmación «yo no formulo hipótesis». Y después la describe como el sello característico de la ciencia experimental. A la ciencia baconiana le interesan los hechos «experimentales» del «mundo real». Pero veamos la primera ley de Newton según aparece en los Principia:

«Todo cuerpo continúa en su estado de reposo o de movimiento uniforme rectilíneo mientras no se vea obligado a cambiar de estado por fuerzas aplicadas sobre él» (Newton, 1971, p. 13).

Comparemos esa afirmación con una de Aristóteles en el libro IV de la Física:

«Un cuerpo, o bien continuaría en su estado de reposo o continuaría necesariamente su movimiento indefinidamente a no ser que una fuerza mayor interfiera con él» (Aristóteles, 1961, 215a20, p. 72).

Las dos descripciones son casi idénticas. Para Newton es una situación idealizada que es útil para definir la función de una fuerza. Para Aristóteles es una descripción de lo que sería el movimiento en la nada si existiera tal nada. Pero tal movimiento no puede observarse, afirma Aristóteles (y Newton asentiría), y por lo tanto no es natural (y Newton no asentiría). Aristóteles concluye su descripción del movimiento como sigue: «O bien, pues, nada tiene un movimiento natural o bien no existe la nada» (Aristóteles, 1961, 215a15 p. 72).

¿Quién es entonces el observador y el realista? ¿Newton, que propone un mundo ideal sin fricción, o Aristóteles, que comenta el mundo tal como es? Con criterio, se ve que la elección de Newton de la propuesta de Galileo de una nada hipotética por encima del rechazo de Aristóteles de una nada física es productivo para el desarrollo de la ciencia. Pero es una distorsión inmensa ver ese desarrollo como una batalla contra las ideas «obviamente falsas» de Aristóteles.

Este ejemplo de mala representación histórica no es un hecho aislado. En el libro de texto moderno abundan afirmaciones sobre los análisis y procedimientos de Newton en los Principia, pero guardan poco parecido con el contenido real de esa obra. Es un libro difícil de leer para el físico moderno y casi ningún físico lo ha abierto siquiera. Sin embargo, al escribir un libro de texto moderno atribuyen a lo Principia elementos extrapolados de los siglos xviII y XIX. Los modernos libros de texto de astronomía atribuyen a Copérnico y Kepler visiones y valores modernos en vez de las interesantes posturas de transición que representan en realidad (Brackenbridge, 1982, p. 117). Ante las contribuciones de Galeno, los biólogos a menudo reaccionan de forma muy parecida a como los físicos reaccionan ante las contribuciones de Aristóteles: afirmando que el largo período en que tales obras fueron autoridad fue perjudicial para el desarrollo de la disciplina. Harvey se presenta como el gran liberador cuya «circulación de la sangre» liberó la disciplina de flujo de mareas «obviamente» incorrecto de Galeno. Pero una lectura atenta de estas fuentes primarias muestra las mismas limitaciones en el caso de Galeno y Harvey que hemos observado en Aristóteles y Galileo.

Además de ser posible (las fuentes primarias existen) sería deseable (la ciencia valora la exactitud) incluir en los libros de texto una historia que no esté 
distorsionada. Pero, ¿procede hacerlo? Como Kuhn ha señalado, «el desprecio del dato histórico está profundamente, y probablemente por motivos funcionales, en la ideología de la profesión científica». Un libro de texto como Introduction into concepts and theories in phisical sciences (Introducción a conceptos y teorías de las ciencias físicas) de Gerald Holton, indica que, además de ser posible, es deseable escribir un libro de texto introductorio que sea exacto históricamente y efectivo pedagógicamente. La primera edición (1952), utilizada principalmente en cursos no científicos, fue ampliada para la segunda edición (1973) con la ayuda de Stephen Brush para utilizarse en cursos avanzados de ciencias. Pero tanto Holton como Brush son físicos reputados que han trabajado durante un tiempo considerable como historiadores de la ciencia. Sin embargo, no existe tiempo ni motivación para que el instructor medio de Física de Instituto o Universidad pueda perseguir tales conocimientos adicionales. Es más, desde el momento en que la gran mayoría de libros de texto cuentan una historia de la Física distinta a la de Holton, ¿con qué criterio elegirá el instructor?

Sugiero que el modo más eficiente y efectivo de empezar a remediar la situación es poner en manos de instructores interesados unas introducciones a un número limitado de fuentes primarias importantes. Armados con cierto conocimiento de primera mano sobre material crítico de autores tales como Aristóteles, Galileo y Newton, los instructores serían capaces de evaluar las posturas de los libros de texto y finalmente producir de su cosecha libros de texto más exactos históricamente. El problema es, por supuesto, que las fuentes primarias tales como las Física de Aristóteles, Las dos nuevas ciencias de Galileo y los Principios de Newton son difíciles de entender aunque existan traducciones. Galileo mismo es consciente de esta dificultad cuando, tras una digresión matemática de veinte páginas sobre el tema principal del Día Uno de Las dos nuevas ciencias, hace que Sagrado (el «joven brillante») exclame lo que sigue:

«Sagrado: Una demostración muy inteligente y elegante, pero así hemos terminado hablando de geometría al discutir las opciones presentadas por Simplicio (el defensor de Aristóteles), objeciones de gran importancia» (Galileo, 1914, 104, p. 60).

Si el lector primerizo, y mucho menos el Sagrado de Galileo, debe pelear con tales digresiones, el interés se desvanecerá mucho antes de alcanzar el tratamiento de la caída libre. Y lo mismo es cierto para la Física de Aristóteles y los Principia de Newton, los cuales son, con buen motivo, «más loados que leídos».

Consciente de esta dificultad y de la necesidad de superarla, el Fondo Nacional para las Humanidades (National Endowment for the Humanities) ha introducido recientemente un programa de apoyo a la preparación de estudios comentados de grandes textos de ciencias. Las metas del programa se establecen como sigue:

«El Fondo pretende prestar apoyo a la preparación de obras orientadas hacia la publicación de guías de estudio de escritos científicos de importancia histórica desde la Antigüedad hasta el siglo XX... Estos volúmenes pretenden hacer accesible al público lector en general y a los alumnos universitarios los escritos científicos del pasado y posibilitar que aquellos con poca o nula experiencia previa en Historia de la Ciencia aprecien, por ejemplo, lo temprano que los científicos formularon preguntas sobre la naturaleza, expresaron conceptos nuevos y desarrollaron respuestas a esas preguntas."

Estas guías de estudio no deben verse como una introducción a la historia de las ideas científicas o como sinopsis de los textos escogidos:

«Una guía de estudio... será un texto de compañía con las, necesarias revisiones, esbozos, diagramas, glosas y explicaciones. Podría hacer elaboraciones a partir del texto, 
hacer explícito a los lectores lo que implican los textos escogidos, explicar conceptos científicos o matemáticos difíciles, comentar el uso de términos importantes, destacar pasajes clave y realizar nuevas traducciones, y de este modo suministrar lo necesario para que estas fuentes primarias se lean con provecho."

Ya existen varios estudios en preparación. Algunos más serán propuestos y algunos de éstos se subvencionarán. La responsabilidad primera del Fondo para las Humanidades es para con el humanista; y a este fin hace que los escritos científicos del pasado estén disponibles y sean inteligibles para aquellos cuyo interés primero es la Historia o la Filosofía. Pero también servirá a la comunidad científica al quedar esas mismas fuentes a su alcance.

\section{CONCLUSION}

Imparto cursos de Física, de Historia de la Ciencia y de materias interdisciplinares. Las metas que me marco a mí mismo en tales cursos son dobles: (1) Hacer llegar a los alumnos de ciencias el conocimiento de la rica herencia cultural e intelectual de su disciplina y (2) hacer llegar a los alumnos de Humanidades la relevancia e importancia de comprender las empresas científicas presentes y pasadas como elementos importantes en la comprensión de otras empresas humanísticas. Mi reto como instructor es persuadir, por una parte, a los alumnos de ciencias de que los conceptos científicos contemporáneos tales como espacio, tiempo, nada, movimiento, etc., no son «obvios» y que su comprensión y apreciación se potencian sabiendo cómo han evolucionado y siguen evolucionando. Por otra parte, el reto es persuadir a los alumnos de humanidades de que los conceptos científicos contemporáneos no son definiciones estériles de interés únicamente para los del laboratorio, sino que están relacionados e interactúan con conceptos utilizados en otros campos de estudio. Para el científico, comprender la dependencia por parte del sistema celeste de Ptolomeo de la teoría platónica del conocimiento es comprender lo mucho que depende de otros elementos culturales la teoría física en vigor; para el humanista, es comprender la viabilidad de los conceptos científicos como índices culturales.

El grueso de mi docencia es de Física. Tan sólo de vez en cuando tengo la oportunidad de ofrecer un curso dedicado a la Historia de la Ciencia. Pero mi comprensión de la historia real de mi disciplina ha potenciado grandemente mi forma de enseñar la ciencia. Se tarda poco más tiempo en presentar a Aristóteles como una mente científica de primer orden que presentarlo incorrectamente como un lastre obvio para el desarrollo de la ciencia. Galileo es más interesante como mente emprendedora capaz de extravagancias y distorsiones, así como de contribuciones y visiones. Para él corregir errores «obvios» es un reto mucho menor que el de crear programas «innovadores». Ver las leyes del movimiento de Newton como parte de una lucha continua de la historia de las ideas es mucho más gratificante que hacerlas aparecer como una contribución solitaria (Brackenbridge, 1988, p. 9). Y las gratificaciones crecen no sólo para el instructor con ideas más claras, sino también para sus colegas docentes y, más importante aún, para sus alumnos. El libro de texto tal como existe ahora es necesario, pero no es suficiente. La excitación y el sabor plenos de la ciencia como proceso creativo no se pueden experimentar en un vacío histórico y filosófico. 


\section{Referencias}

Aristotle (1961). Physics (Richard Hope, trans.). Lincoln: University of Nebraska Press.

BraCKEnRIDGE, J. B. (1982). Kepler, elliptical orbits, and celestial circularity: A study in the persistence of metaphisical commitment. Annals of Science, 117-143; 265-295.

BRACKENRIDGE, J. B. (1985). Kuhn, paradigms, and astronomy: Astronomy as a case study of Kuhnian paradigms. Proceedings of the American Pbilosophical Society, 129, 433-455.

BrACKENRIDGE, J. B. (1988). Newton's mature dynamics: Revolutionary or reactionary. Annals of Science.

CoOper, L. (1936). Aristotle, Galileo, and the Tower of Pisa. Ithaca: Cornell University Press.

Galileo (1914). The two news sciences (H. Crew y A. De Salvio, trans.). Nueva York: Dover.

Galileo (1974). The two new sciences (Stillman Drake, trans.). Madison: The University of Wisconsin Press.

Hotron, G. (1952). Introduction to concepts and theories in physical sciences (1st.ed.) Cambridge: Addison-Wesley.

Holton, G., y Brusn, S. (1973). Introduction to concepts and theories in physical sciences (2nd ed.). Reading: Addision-Wesley.

Kunn, T. (1957). The Copernican revolution. Cambridge: Harvard University Press.

Kunn, T. (1970). The structure of scientific revolutions (2nd ed.). Chicago: The University of Chicago Press.

Kurin, T. (1977). The essential tension. Chicago: The University of Chicago Press.

$\mathrm{N}_{\mathrm{Ew}} \mathrm{TON}, \mathrm{I}$. (1971). The mathematical principles of natural philosopby. Berkeley: University of Ca. lifornia Press.

La educación de las ciencias, la Historia de la Ciencia y el libro de texto, las condiciones necesarias contra las suficientes.

J. Bruce Brackenbridge.

CL\&E, 1991, 11-12, pp. 157-168

Datos sobre el autor: Bruce Brackenbridge es profesor de física en la Universidad de Lawrence, Wisconsin. Sus últimas publicaciones tratan de la mecánica de Newton y la astronomía de Kepler.

Dirección: Universidad de Lawrence. Departamento de Física. Appleton, Wisconsin. 54 192 USA.

Artículo original: Education in Science, History of Science, and the Textbook - Necessary vs. Sufficiente Conditions. En Interchange, 1989; Vol. 20, N. ${ }^{\circ} 2$; pp. 71-80. Reproducido con autorización. Traducción de Eduardo Braun.

(c) De todos los artículos. Deberá solicitarse por escrito autorización de CL\&E y de los autores para el uso en forma de facsímil, fotocopia o cualquier otro medio de reproducción impresa. CL\&E se reserva el derecho de interponer las acciones legales necesarias en aquellos casos en que se contravenga la ley de derechos de autor. 